\title{
UTILIDAD DE LA VALORACIÓN GERIÁTRICA INTEGRAL EN LA EVALUACIÓN DE LA SALUD DEL ADULTO MAYOR
}

\author{
Miguel Gálvez-Cano1,2,a, Helver Chávez-Jimeno ${ }^{1,2, a, b}$, Elizabeth Aliaga-Diaz',2,a
}

\begin{abstract}
RESUMEN
Los adultos mayores conforman un grupo poblacional heterogéneo que, usualmente, presenta una gran carga de enfermedad, pluripatología y, en muchas ocasiones, condiciones subclínicas que comprometen su salud y calidad de vida. Además del componente físico, la salud de los adultos mayores está influenciada, de manera importante, por factores mentales, que incluyen componentes cognitivos y afectivos; factores sociofamiliares, como el abandono; y factores funcionales, como la capacidad para realizar las actividades cotidianas. En respuesta a este escenario complejo, la valoración geriátrica integral se constituye en una herramienta diagnóstica, multidimensional e interdisciplinaria, cuya característica es evaluar la salud del adulto mayor en toda su complejidad, es decir, toma en consideración los aspectos físicos, mentales, sociofamiliares y funcionales para tener un conocimiento completo del estado de salud de la persona mayor $y$, de esta manera, realizar un plan de trabajo con intervenciones apropiadas e individualizadas, tomando en cuenta sus preferencias, sus valores y los de la familia.
\end{abstract}

Palabras clave: Adulto mayor; Evaluación geriátrica; Atención integral de salud (fuente: DeCS BIREME).

\section{USEFULNESS OF THE COMPREHENSIVE GERIATRIC ASSESSMENT FOR EVALUATING THE HEALTH OF OLDER ADULTS}

\begin{abstract}
Older adults comprise a heterogeneous population group that usually has a high disease burden, comorbidities, and, in many cases, subclinical conditions that compromise their health and quality of life. In addition to the physical component, the health conditions of elderly individuals are significantly influenced by cognitive and affective components, social and family factors such as abandonment, and functional factors including the ability to perform everyday activities. In response to this complex scenario, the comprehensive geriatric evaluation constitutes a multidimensional and interdisciplinary diagnostic tool that assesses the health of older adults in all of its complexity by considering the physical, mental, social/family, and functional needs to obtain full knowledge of older person's health status and creating a plan that consists of appropriate and individualized interventions that considers the preferences and values of older individuals and their families.
\end{abstract}

Keywords: Aged; Geriatric assessment; Comprehensive health care (source: MeSH NLM)..

\section{INTRODUCCIÓN}

El estado de salud de los adultos mayores depende de diversos factores (Figura 1): la salud física, el estado cognoscitivo, el estado de ánimo y las condiciones sociofamiliares. Cabe señalar que estos factores cobran mayor importancia en los adultos mayores que en los adultos jóvenes, debido a que las enfermedades crónicas y degenerativas son más prevalentes en los mayores (1). Por ejemplo, alteraciones del estado mental, como la demencia, son mucho más frecuentes en este grupo poblacional, las que al asociarse con otras enfermedades crónicas, como diabetes, hipertensión arterial, osteoartritis, degeneración macular y algunas de ellas oligoasintomáticas o subclínicas, propias del envejecimiento, conllevan un mayor deterioro del estado de salud, que no solo afecta el componente físico, sino que acarrean una situación de dependencia. Estas enfermedades, aunadas a condiciones sociofamiliares desfavorables por cuidados inapropiados, pueden empeorar la salud física, generar cambios en el estado de ánimo o llevar a situaciones de mayor dependencia física. Por otro lado, dado que, generalmente, las personas mayores no perciben una pensión o no están en capacidad de trabajar, pasan a depender económicamente de sus familiares, generándoles inseguridad y temor, y ocasionándoles alteraciones anímicas y conflictos familiares, los que, a su vez, repercuten en su salud.

\footnotetext{
Instituto de Gerontología, Universidad Peruana Cayetano Heredia. Lima, Perú.

Hospital Nacional Cayetano Heredia. Lima, Perú.

Médico geriatra; ${ }^{\mathrm{b}}$ médico internista

Recibido: 07/03/2016 Aprobado: 01/06/2016
}

Citar como: Gálvez-Cano M, Chávez-Jimeno H, Aliaga-Diaz E. Utilidad de la valoración geriátrica integral en la evaluación de la salud del adulto mayor. Rev Peru Med Exp Salud Publica. 2016;33(2):321-7. doi: 10.17843/rpmesp.2016.332.2204 


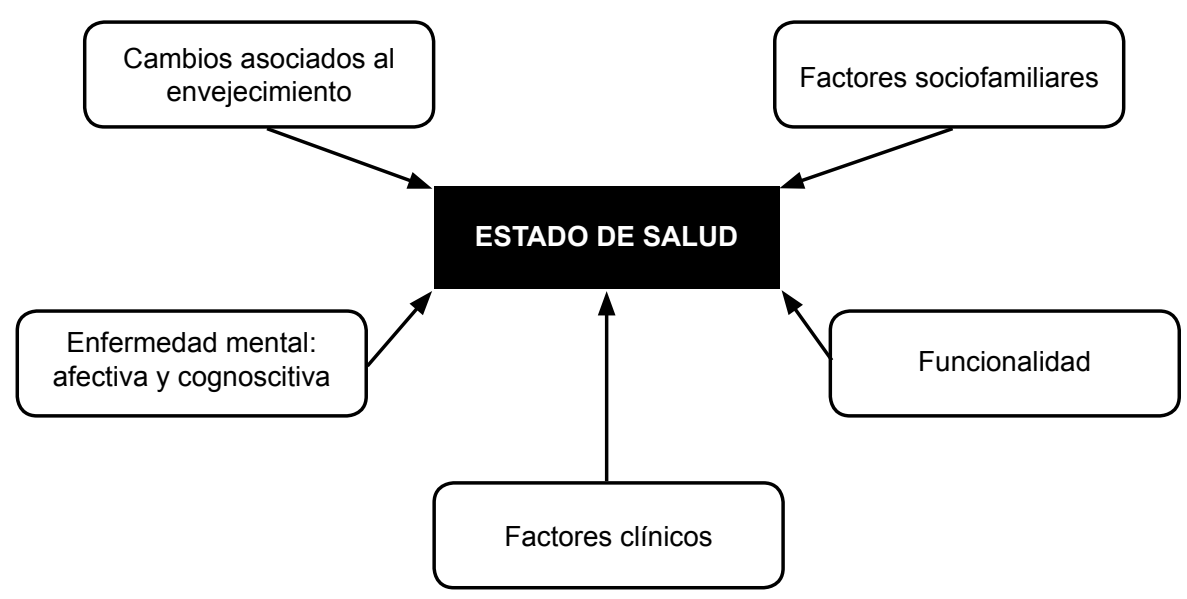

Figura 1. Factores que influyen en el estado de salud del adulto mayor

El segmento poblacional que comprende a las personas mayores es heterogéneo, personas de 90 años pueden estar saludables y personas de 65 años estar muy enfermas; personas con demencia o sin demencia; personas activas que siguen laborando y otras postradas o dependientes.

Finalmente, es en el grupo de adultos mayores donde con mayor frecuencia ocurren las presentaciones atípicas de las enfermedades, lo que dificulta su reconocimiento. Por ello, en respuesta a estas características -población heterogénea, diversas y complejas variables que influyen en su salud, presentación atípica de las enfermedadessurge la valoración geriátrica integral, una evaluación fundamentalmente individualizada que toma en cuenta los diversos factores que afectan el estado de salud para obtener una visión completa del adulto mayor.

El propósito de este artículo es presentar esta herramienta útil de evaluación, que aunque es indispensable para el uso de los médicos geriatras, también sea conocida y utilizada por diversos profesionales de la salud que atienden a estas personas, en especial a los profesionales del primer nivel de atención, que son el primer contacto del adulto mayor con el sistema de salud.

\section{DEFINICIÓN DE EVALUACIÓN GERIÁTRICA INTEGRAL}

La valoración geriátrica integral (VGI) se define como "un proceso diagnóstico evolutivo multidimensional e interdisciplinario, diseñado para identificar y cuantificar los problemas físicos, funcionales, psíquicos y sociales que puedan presentar los adultos mayores, con el propósito de desarrollar un plan individualizado de cuidados integrales, que permita una acción preventiva, terapéutica, rehabilitadora y de seguimiento, con la óptima utilización de recursos, a fin de lograr el mayor grado de autonomía y mejorar su calidad de vida" (2).
Multidimensional, porque incluye la evaluación de varias dimensiones que determinan el estado de salud (funcional, afectiva, cognitiva, social y física), e interdisciplinario, porque requiere del aporte de varios profesionales para la evaluación y manejo de los problemas de los adultos mayores. El equipo de salud básico encargado de la evaluación y cuidado de las personas mayores debería estar conformado por un médico, un enfermero, un profesional de servicio social y un terapista físico.

Solo con una evaluación completa (integral) es posible la identificación cabal del estado de salud de la persona mayor, usualmente compleja y en ocasiones no fácil de determinar, con el fin de conocer el deterioro de la salud y sus causas, para brindarle una atención diferenciada, según lo que el paciente requiera y desee, pero también identificando los aspectos positivos que pueda tener o contar la persona o su familia para superar o manejar las adversidades y enfermedades y así poder enfrentar las dificultades y adaptarse a las condiciones limitantes que se presenten. Con el objetivo de mejorar su calidad de vida se deben establecer las prioridades de intervención para desarrollar un plan de trabajo apropiado y de acuerdo a los intereses del adulto mayor y de su familia.

La VGI cobra mayor importancia en las personas con pluripatología, dependientes, con problemas de salud complejos y frágiles, debido a que identifica mejor sus riesgos, sus déficits, y permite una intervención apropiada ${ }^{(3)}$.

Finalmente podemos concluir que la VGI nos permite identificar y priorizar los aspectos negativos y positivos de la salud de las personas mayores, con el fin, no solo de prevenir y tratar la enfermedad y rehabilitar la discapacidad, sino impulsar un envejecimiento activo y saludable y que la experiencia de ser mayor sea positiva, es decir, que sea posible lograr un envejecimiento exitoso. 


\section{CONOCIMIENTO DEL ESTADO DE SALUD DEL ADULTO MAYOR}

El conocimiento completo del estado de salud y de los factores que lo determinan se realiza al evaluar los siguientes componentes: el mental, que incluye el componente cognoscitivo y anímico; el clínico, que incluye el estado físico; el sociofamiliar, que comprende el apoyo familiar, social y económico; y el funcional (Tabla 1).

\section{COMPONENTE FUNCIONAL}

La funcionalidad es la capacidad que tiene la persona de valerse por sí misma en las actividades de la vida diaria. Mide la capacidad de realizar las actividades cotidianas básicas que le permiten subsistir, tales como comer, vestirse, levantarse, bañarse, manejar sus excretas $(2,3)$. También mide las actividades instrumentales de la vida diaria, es decir, evalúa si la persona puede vivir sola sin ayuda, si puede preparar sus comidas, usar

Tabla 1. Componentes de la valoración geriátrica integral

\begin{tabular}{|c|c|}
\hline Componente & Instrumento \\
\hline $\begin{array}{l}\text { FUNCIONAL } \\
\text { Evalúa la función } \\
\text { del adulto mayor, } \\
\text { determinando su } \\
\text { capacidad para realizar } \\
\text { actividades básicas, } \\
\text { instrumentales y } \\
\text { avanzadas de la vida } \\
\text { diaria }^{(4)} \text {. }\end{array}$ & $\begin{array}{l}\text { - Evaluación del equilibrio y la } \\
\text { marcha: pruebas de Get up } \\
\text { and Go }{ }^{(5)} \text { y Tinetti }{ }^{(6)} \text {. } \\
\text { - Índice de Katz }{ }^{(7)} \text {. } \\
\text { - Índice de Barthel }{ }^{(4)} \text {. } \\
\text { - Índice de Lawton y Brody }{ }^{(4)} \text {. } \\
\text { - Escala Física de las actividades } \\
\text { avanzadas de Reuben }{ }^{(4)} \text {. }\end{array}$ \\
\hline $\begin{array}{l}\text { MENTAL } \\
\text { Cognoscitivo: evalúa el } \\
\text { deterioro neurocognitivo } \\
\text { mayor y menor }{ }^{(8)} \text {. } \\
\text { Afectivo: evalúa el } \\
\text { ánimo, centrándose en la } \\
\text { depresión y ansiedad. }\end{array}$ & $\begin{array}{l}\text { - Test de Pfeiffer (9). } \\
\text { - Minimental de Folstein }{ }^{(4)} \text {. } \\
\text { - Escala de depresión de } \\
\text { Yesavage }{ }^{(10)} \text {. } \\
\text { - Escala reducida de cuatro } \\
\text { preguntas para evaluar } \\
\text { depresión (GDS4) }{ }^{(11)}\end{array}$ \\
\hline $\begin{array}{l}\text { CLíNICo } \\
\text { Evalúa el estado físico } \\
\text { del adulto mayor, } \\
\text { comprende la evaluación } \\
\text { médica tradicional, que } \\
\text { incluye la anamnesis y } \\
\text { examen físico, y pruebas } \\
\text { de apoyo diagnóstico }{ }^{(3)} \text {. }\end{array}$ & $\begin{array}{l}\text { - Historia clínica tradicional. } \\
\text { - Pruebas de apoyo al } \\
\text { diagnóstico: pruebas analíticas } \\
\text { y de imágenes. } \\
\text { - Valoración nutricional. }\end{array}$ \\
\hline $\begin{array}{l}\text { SOCIOFAMILIAR } \\
\text { Evalúa fundamentalmente } \\
\text { el estado sociofamiliar } \\
\text { y económico, pero } \\
\text { también debe incluir las } \\
\text { condiciones ambientales y } \\
\text { la red de apoyo social }{ }^{(3,4)} \text {. }\end{array}$ & $\begin{array}{l}\text { - Escala de valoración sociofamiliar } \\
\text { la modificada por Merino }{ }^{(12)} \text {. }\end{array}$ \\
\hline
\end{tabular}

el teléfono y los medios de transporte, hacer compras, ser responsable con su medicación, en el manejo de sus asuntos económicos y en el cuidado de la casa; también evalúa actividades avanzadas de la vida diaria, y si puede participar en las actividades de su comunidad. El deterioro de la funcionalidad es poco frecuente en personas jóvenes, no así en personas mayores que debido a la ocurrencia de diversas enfermedades crónicas y degenerativas y a los cambios neuromusculares asociados al envejecimiento pueden ver alterada su funcionalidad.

En la evaluación de este componente se usan diferentes escalas, entre las pruebas que se usan para evaluar el desempeño en realizar las actividades básicas de la vida diaria (ABVD) se encuentra el índice de Katz que mide diversas funciones como el alimentarse, la continencia, levantarse, ir al baño, vestirse, y bañarse. Las categorías van desde la independencia (categoría A) hasta la dependencia total (categoría G, dependiente para todas las funciones), los estados intermedios son dependencia parcial.

La evaluación de la funcionalidad no solo nos permite determinar el nivel de deterioro, sino también las causas que lo originaron, con el objeto de intervenir para revertir o evitar un mayor deterioro; asimismo, permite indicar el cuidado apropiado, por ejemplo, si una persona no puede bañarse y vestirse sola, porque se cae o tiene una alteración del equilibrio y de la marcha, se debería asignar una persona para que la ayude a realizar estas actividades o incluso se encargue por completo de estas. Si la evaluación de la funcionalidad de una persona determina que no es capaz de realizar las actividades básicas que permiten su subsistencia, como alimentarse, levantarse de la cama, asearse, es urgente asignar una persona para que la asista, ya que esta alteración puede comprometer su salud y su vida.

La evaluación de la funcionalidad es importante también para la toma de decisiones acerca de los cuidados e intervenciones en las personas mayores. Por ejemplo, una persona de 90 años que se vale por sí misma, que hace sus compras y sale a pasear y sufre una fractura de cadera por una caída, debido a que su estado funcional previo es bueno, es apropiado que sea operada. Por otro lado, si a una persona de 65 años que por su estado de salud permanece postrada permanentemente y necesita ayuda para alimentarse, bañarse y vestirse se le descubre una neoplasia de pulmón, debido a su estado funcional previo tendrá menor opción de recibir un tratamiento oncológico completo.

La funcionalidad permite también evaluar la severidad de la enfermedad. Por ejemplo, si una persona mayor tiene una neumonía y continúa valiéndose por sí misma, 
probablemente esta no sea severa; pero si la neumonía le impide vestirse, levantarse o comer sola porque está desorientada o porque le falta el aire, esta alteración de la funcionalidad implicaría una mayor severidad de la infección y, por lo tanto, habría que someterla a un manejo intrahospitalario.

Las alteraciones en la funcionalidad también nos permiten identificar algunas enfermedades que se presentan sutilmente o de forma atípica. Por ejemplo, si una persona de 80 años que hasta hace un año se valía por sí misma y en la actualidad no puede bañarse ni vestirse sola y tiene dificultad para levantarse, habría que preguntarse si estos cambios son propios del envejecimiento o de alguna enfermedad subyacente no identificada, como enfermedad de Parkinson, osteoartritis de rodilla, o deterioro cognitivo, ya que no siempre son fáciles de detectar, pero se pueden sospechar a partir de cambios en la funcionalidad, y así poder determinar las medidas a implementar para revertir o aminorar el deterioro.

La funcionalidad también es un determinante importante de la calidad de vida ${ }^{(4,13)}$. Cuando se consulta a las personas mayores sobre su percepción de los factores más importantes respecto a la calidad de vida, así como es importante mencionar la ausencia del dolor, también es importante incluir factores como valerse por sí mismos y no sentirse una carga para su familia. Por ello, al evaluar la funcionalidad, prevenirla y manejarla también estamos contribuyendo a mejorar la calidad de vida de los adultos mayores y de sus familiares.

Finalmente, la funcionalidad o la capacidad para valerse por sí mismo es la resultante de todos los sistemas y órganos del cuerpo humano. Si una persona es capaz de valerse por sí misma o tiene la capacidad de vivir sola de manera independiente, entonces su cerebro, su pulmón y su corazón estarán funcionando adecuadamente. Por ello, en toda evaluación integral de los adultos mayores siempre se debe incluir la evaluación de la funcionalidad.

\section{SIINDROMES GERIÁTRICOS}

Un síndrome geriátrico es una entidad que ocurre frecuentemente en personas mayores y representa algún problema de salud o enfermedad que, si bien aun no se manifiesta abiertamente, ya implica una patología o un riesgo para su salud. Por ejemplo, las caídas, una condición frecuente en las personas mayores (aproximadamente 1 de cada 2 adultos mayores se cae 1 vez al año $\left.{ }^{(14)}\right)$ pueden ser la manifestación de enfermedad de Parkinson, de osteoartritis, de demencia o de problemas visuales o de una combinación de todas ellas que, por presentarse de manera sutil, pueden pasar desapercibidas, pero que al progresar o al complementarse con otras, alteran la marcha y el equilibrio ocasionando las caídas, las que pueden causar fracturas -como las de cadera- y comprometer la funcionalidad e, incluso, causar la muerte de la persona. Por ello, en la evaluación geriátrica integral se debe incluir un listado de síndromes y problemas geriátricos para evaluar algunas enfermedades que suelen manifestarse de manera atípica, sutil, o a través de estos síndromes geriátricos (Tabla 2).

Las características del síndrome geriátrico son: su origen es de naturaleza compleja, es decir, proviene de múltiples factores que no solo confluyen, sino que se potencian entre ellos ${ }^{(15)}$; suele comprometer diversos órganos y sistemas; su forma de presentación muchas veces no tiene relación con la patología que lo genera $\mathrm{y}$, por lo general, se presenta en sujetos frágiles. Por ejemplo, el delirio, usualmente multifactorial, suele ser la conjunción de factores precipitantes en un persona con condiciones predisponentes ${ }^{(16)}$; entre estas pueden estar el deterioro cognitivo, el uso de psicofármacos y la disfunción renal leve, que ante la ocurrencia de un factor precipitante, como una infección urinaria, que a su vez puede generar alteraciones metabólicas como deshidratación, hiponatremia o mayor azoemia, que junto a la fiebre, el dolor o las molestias propias de la infección urinaria contribuyen, en conjunto, a la

Tabla 2. Principales síndromes geriátricos

\begin{tabular}{ll}
\hline Síndrome geriátrico & \multicolumn{1}{c}{ Definición } \\
\hline Caídas & $\begin{array}{l}\text { Se considera síndrome de caídas cuando ocurren más de dos caídas en 1 año, o un episodio de caída } \\
\text { con complicaciones }{ }^{(2)} \text {. }\end{array}$ \\
\hline Delirio & $\begin{array}{l}\text { Deterioro global de las funciones cognitivas, presentando un comienzo agudo, curso fluctuante con } \\
\text { agitación y alteración del ciclo sueño vigilia }{ }^{(2,3,16)} .\end{array}$ \\
\hline Inmovilismo & $\begin{array}{l}\text { Descenso de la capacidad para desempeñar actividades de la vida diaria por deterioro de las funciones } \\
\text { motoras, lo que lleva a situación de postración }{ }^{(2,4)} \text {. }\end{array}$ \\
\hline Incontinencia & $\begin{array}{l}\text { Pérdida involuntaria de orina, que es objetivamente demostrable y que constituye un problema social e } \\
\text { higiénico. La fecal como expulsión involuntaria de heces }{ }^{(2,4)} .\end{array}$ \\
\hline Fragilidad & $\begin{array}{l}\text { Síndrome biológico de vulnerabilidad al cual se llega por el declive acumulado de los mecanismos } \\
\text { fisiológicos, con pérdida progresiva de la capacidad homeostática, originando que el paciente no pueda } \\
\text { enfrentar condiciones estresantes como infecciones, traumatismos, hospitalizaciones, entre otras }{ }^{(17)} .\end{array}$ \\
\hline
\end{tabular}


presentación de este síndrome. El delirio no suele afectar solo el sistema neurológico, en el caso de la infección urinaria puede afectar el sistema renal (generando azoemia), el osteomuscular (puede generar caídas y postración), el cardiovascular (puede ocasionar arritmias y descompensación hemodinámica). El síntoma de una infección urinaria (trastorno urológico) es desorientación y agitación (trastorno neurológico), lo cual hace que la identificación de la entidad que origina esta alteración sea más compleja por su naturaleza atípica.

Existen múltiples síndromes geriátricos como caídas, delirio, inmovilismo, incontinencia y fragilidad. Pese a que el síndrome de fragilidad tiene una conceptualización ya definida, su diagnóstico clínico es complejo, por ello se han desarrollado diversos marcadores de esta entidad, entre ellos el de la velocidad de la marcha que es el más usado y tiene buena correlación, por ello, sugerimos que se utilice este método para su tamizaje.

Finalmente, los síndromes geriátricos como el delirio suelen ocurrir básicamente en sujetos frágiles, quienes por su poca reserva funcional y su poca capacidad para adaptarse son los que van a presentar de manera atípica y con mayor complejidad y severidad la enfermedad. En el caso de la infección urinaria, esta se presentará como delirio y estará acompañada de trastornos metabólicos como deshidratación, azoemia, caídas y postración e hipotensión asociada a sepsis, pero sin manifestación de síntomas urinarios bajos.

Existen otras entidades también frecuentes en los adultos mayores que no tienen la complejidad de los síndromes geriátricos, los denominados problemas geriátricos, que no resultan necesariamente de la interacción de diversas patologías o problemas de salud o se refieren a un área o situación específica y no siempre se presentan en sujetos frágiles. Por ejemplo, el edentulismo, la deprivación visual y el prostatismo, los que también deben ser evaluados porque afectan la calidad de vida y pueden comprometer la salud del adulto mayor. Estos problemas geriátricos no suelen ser reportados por el paciente o la familia, ya que no le dan la debida importancia o los consideran parte del proceso normal del envejecimiento, pero que en muchos casos pueden manejarse o evitar sus complicaciones ${ }^{3}$.

\section{COMPONENTE SOCIOFAMILIAR}

Otro aspecto importante que define el estado de salud de las personas mayores es conocer el entorno sociofamiliar en el que viven. Pueden existir entornos favorables que promueven un envejecimiento saludable o entornos de abuso, de sobreprotección o de cuidado inapropiado por desconocimiento o por falta de interés. En pediatría se asume que el médico interactúe básicamente con la familia; en geriatría esto se suele dar en algunos casos, en pacientes con demencia o con dependencia funcional, pero se debe tomar en cuenta, siempre que sea posible, la opinión y las preferencias del paciente y evitar la generación de entornos que limiten el desarrollo de las personas mayores ${ }^{(2,3)}$. Por ello es importante conocer el estado sociofamiliar para realizar una intervención individualizada y apropiada. Por ejemplo, si tenemos un adulto mayor con una disfagia orofaríngea severa, asociada a una hemiplejia, consecuencia de una enfermedad cerebro vascular, podemos estabilizar al paciente en la parte cardiovascular, pero si no se trabaja adecuadamente con la familia en relación a la alimentación y la prevención de neumonías aspirativas, el paciente probablemente se va a complicar.

Existen diferentes escalas para la evaluación del componente sociofamiliar, ninguno de ellas validada para nuestra población, nosotros recomendamos la escala de valoración sociofamiliar (de Gijón) modificada por Merino que tiene dos categorías el entorno social adecuado y el riesgo y problema social.

\section{USO DE ESCALAS}

Respecto al uso de escalas en la valoración geriátrica integral, es importante señalar que su uso nos permite sistematizar la información unificando criterios y diagnósticos; evaluar de manera objetiva los problemas o las condiciones que presentan las personas mayores, permitiendo un adecuado seguimiento; evaluar nuestra intervención y acortar el tiempo de evaluación, ya que su uso como tamizaje rápidamente nos puede dar una idea general y completa del estado de salud de las personas. Por ejemplo, el índice de Katz, que mide la funcionalidad a través de la capacidad para realizar las actividades básicas de la vida diaria (bañarse, vestirse, usar el retrete, levantarse, alimentarse). Un paciente Katz A es una persona independiente, al referirnos a él como Katz A, cualquier profesional, en cualquier sistema de salud, va a tener el mismo concepto de esta persona, es decir, que es independiente para realizar las actividades básicas de la vida diaria; de esta manera, la evaluación se estandariza. En el caso de que en un año pierda la independencia para bañarse y vestirse, es decir, se torne Katz C, indicaría, también de manera objetiva, que hay un deterioro de su funcionalidad y que en el caso de que se hubiera hecho una intervención preventiva, esta no habría sido efectiva.

Finalmente, debido a que la evaluación geriátrica integral evalúa varios dominios, esta puede demorar en su realización, con el uso de escalas cortas y validadas se puede realizar una evaluación completa y eficiente sin implicar un tiempo prolongado. 


\section{PLAN DE TRABAJO E INTERVENCIÓN APROPIADA}

Luego de la evaluación, con toda la información disponible de los diferentes aspectos que influyen en la salud del adulto mayor, se debe diseñar un plan de trabajo, objetivo práctico de la valoración geriátrica integral.

Dada la complejidad y multiplicidad de los factores que intervienen en el estado de salud de las personas mayores, es importante priorizar qué factores o enfermedades se van a intervenir primero; usualmente serán los que generen un mayor compromiso del estado de salud o sea el factor desencadenante, o los que afecten en mayor medida su calidad de vida y supervivencia. Es importante que en esta priorización se tomen en cuenta las preferencias y valores del paciente y de su familia y que se brinde un espacio especial a la queja principal o motivo de la consulta. Por esta razón, en muchas ocasiones algunas intervenciones podrán ser diferidas hasta conseguir la estabilidad del paciente o realizar las actividades con mayor impacto en la calidad de vida.

Otro aspecto importante de la intervención es explicar al paciente y a la familia el estado de la salud de la persona, los factores que influyen positiva y negativamente en este y la naturaleza de la intervención, con el fin de unificar esfuerzos y promover la colaboración del paciente y de su familia.

En el caso de que el paciente tenga alguna condición terminal, como demencia severa, enfermedad pulmonar avanzada o cáncer metastásico, es importante incluir en el plan de trabajo las denominadas decisiones avanzadas. Estas se definen como las preferencias del paciente o su familia en el alcance y tipo de cuidados al final de la vida; por ejemplo, en el caso de un paciente con fibrosis pulmonar avanzada, la persona puede optar por pasar los últimos días de su vida en casa, y en el caso de no poder alimentarse, preferir la sedación a la colocación de una sonda nasogástrica. Las sugerencias para la toma de estas decisiones se deben dar en momentos de estabilidad, ya que en las crisis no siempre se toman buenas decisiones, pero el factor más importante para tomar una buena decisión es tener una adecuada información. Por ello, es vital brindar, al paciente y a la familia, la información más precisa y en el momento adecuado ${ }^{(4)}$.

En muchos casos el paciente mayor va a necesitar cuidados continuos de parte de sus familiares; estos cuidados generan una gran carga, no solo física, sino también emocional y económica. Por ello, es importante que en el plan de trabajo se considere ayudar a la familia a que se organice adecuadamente para el cuidado de su familiar y así evitar el desgaste o el colapso del o las personas encargadas de su cuidado.

La valoración geriátrica integral, en el caso del paciente hospitalizado que va ser dado de alta, nos permite planificar adecuadamente sus cuidados posteriores, orientar al paciente y a la familia, e informar al médico de cabecera o de atención primaria sobre lo que se ha realizado y el trabajo pendiente.

La valoración geriátrica integral nos brinda la información necesaria para realizar correctamente estas intervenciones. Por ejemplo, si el paciente tiene una demencia severa, básicamente interactuaremos y orientaremos a la familia; debemos conocer el estado funcional de la persona para que la familia organice los cuidados y no se genere un desgaste inapropiado. Esto implica conocer a la familia, sus fortalezas y debilidades, para ofrecerle la información pertinente y entrenarla en el cuidado de su familiar, y que de acuerdo a sus preferencias se definan los objetivos del cuidado, tanto en el aspecto de las decisiones avanzadas, como sobre el manejo de las patologías médicas, ya que serán los familiares quienes, bajo la orientación médica, decidirán qué se va a priorizar: el mantenimiento de la calidad de vida o prolongar la vida.

Finalmente, la valoración geriátrica integral no debe ser solo un listado de problemas, riesgos y déficits; necesariamente debe incluir y promover los aspectos positivos de la salud de la persona mayor, no solo en el ámbito físico, como darle la seguridad y confianza de que no tiene una enfermedad física grave o incapacitante y que, por tanto, sus problemas no deberían afectar su calidad de vida y que puede ser una persona útil a su familia y a su comunidad, sino también los aspectos mentales y anímicos, que son fortalezas que pueden ayudar decisivamente a la prevención o a la recuperación del daño. Se debe resaltar que cuando se dispone de buenas condiciones familiares y de actividad, de ocio o tiempo libre, de ayuda a la familia o voluntariado e incluso laborales, estas van a permitir que la persona mayor disfrute de su tiempo y experimente un envejecimiento activo, saludable; incluso, exitoso.

Contribuciones de autoría: MGC, HCJ Y EAD han participado en la concepción del artículo. EAD y MGC realizaron la redacción del artículo. HCJ y MGC hicieron revisión crítica del artículo.

Fuentes de financiamiento: autofinanciado.

Conflictos de interés: los autores declaran no tener conflictos de interés. 


\section{REFERENCIAS BIBLIOGRÁFICAS}

1. Velásquez A, Cachay C, Munayco C, Poquioma E, Espinoza R, Seclén Y. La carga de enfermedad y lesiones en el Perú. Lima: MINSA; 2009.

2. Varela L. Valoración geriátrica integral y síndromes geriátricos. En: Varela L, editor. Principios de geriatría y gerontología. $2^{\mathrm{a}}$ ed. Lima: UPCH; 2011. p. 195-207.

3. Howard M, Kenneth R, Kenneth W. Brocklehurst's Textbook of Geriatric Medicine and Gerontology. $7^{\text {th }}$ ed. Philadelphia: Editorial Saunders Elseiver. 2010.

4. Abizanda Soler P, Gómez-Pavón J, Martín-Lesende I, Baztán Cortés JJ. Detección y prevención de la fragilidad: una nueva perspectiva de prevención de la dependencia en las personas mayores. Med Clin (Barc). 2010;135(15):71319. doi: 10.1016/j.medcli.2009.04.028

5. Mathias S, Nayak US, Isaacs B. Balance in elderly patients: the "get-up and go" test. Arch Phys Med Rehabil. 1986;67(6):387-9.

6. Tinetti ME. Performance-oriented assessment of mobility problems in elderly patients. J Am Geriatr Soc. 1986;34(2):119-26.

7. Katz PR, Dube DH, Calkins E. Use of a structured functional assessment format in a geriatric consultative service. J Am Geriatr Soc. 1985;33(10):681-
6. doi: $10.1111 / \mathrm{j} .1532-5415.1985$. tb01776.x

8. Asociación Americana de Psiquiatría. Manual diagnóstico y estadístico de trastornos mentales. $5^{\text {a }}$ ed. Virginia: Asociación Americana de Psiquiatría; 2014.

9. Pfeiffer E. A short portable mental status questionnaire for the assessment of organic brain deficit in elderly patient: J Am Geriatr Soc. 1975;23(10):433-41. doi: 10.1111/ j.1532-5415.1975.tb00927.x

10. Yesavage JA, Brink TL, Rose TL, Lum O, Huang V, Adey $\mathrm{M}$, et al. Development and validation of a geriatric depression screening scale: a preliminary report. J Psychiatr Res. 1982;17(1):37-49.

11. De la Torre J, Shimabukuro R, Varela L, Krüger H, Huayanay L, Cieza J. et al. Validación de la versión reducida de la escala de depresión geriátrica en el consultorio externo de geriatría del Hospital Nacional Cayetano Heredia. Acta Med Per. 2006;23(3):144-7.

12. Merino R, Varela L, Manrique G. Evaluación del paciente geriátrico hospitalizado. Orientado por problemas: Estudio prospectivo de 71 casos. Rev Med Hered. 1992;3(2):51-9.

13. Ferrer A, Formiga F, Almeda J, Alonso J, Brotons C, Pujol R. Calidad de vida en nonagenarios: género, funcionalidad, y riesgo nutricional como factores asociados. Med Clin (Barc). 2010;134(7):303-6. doi: 10.1016/j.medcli.2009.06.070

14. Rubenstein LZ, Josephson KR. The epidemiology of falls and syncope. Clin Geriatr Med. 2002;18(2):141-58.

15. Inouye SK, Studenski S, Tinetti ME. Kuchel GA. Geriatric syndromes: clinical, research, and policy implications of a core geriatric concept. J Am Geriatr Soc. 55(5):780-91. doi: 10.1111/j.15325415.2007.01156.x

16. Inouye SK. Delirium in Older Persons. N Engl J Med. 2006;354(11):1157-65. doi: 10.1056/NEJMra052321

17. Lesende I. Baztán J. Gorroñogoitia A. Abizanda P. Gómez J. Detección y manejo de la fragilidad en atención primaria; actualización. Buenos Aires: Sociedad Iberoamericana de Información Científica (SIIC salud); 2012.

Correspondencia: Miguel Gálvez Cano Dirección: Avenida Morro Solar 480, Departamento 201, Urbanización Santa Teresa, Surco, Lima 33, Perú.

Teléfono: (+511) 997365249

Correo electrónico:miguel.galvez.c@upch.pe

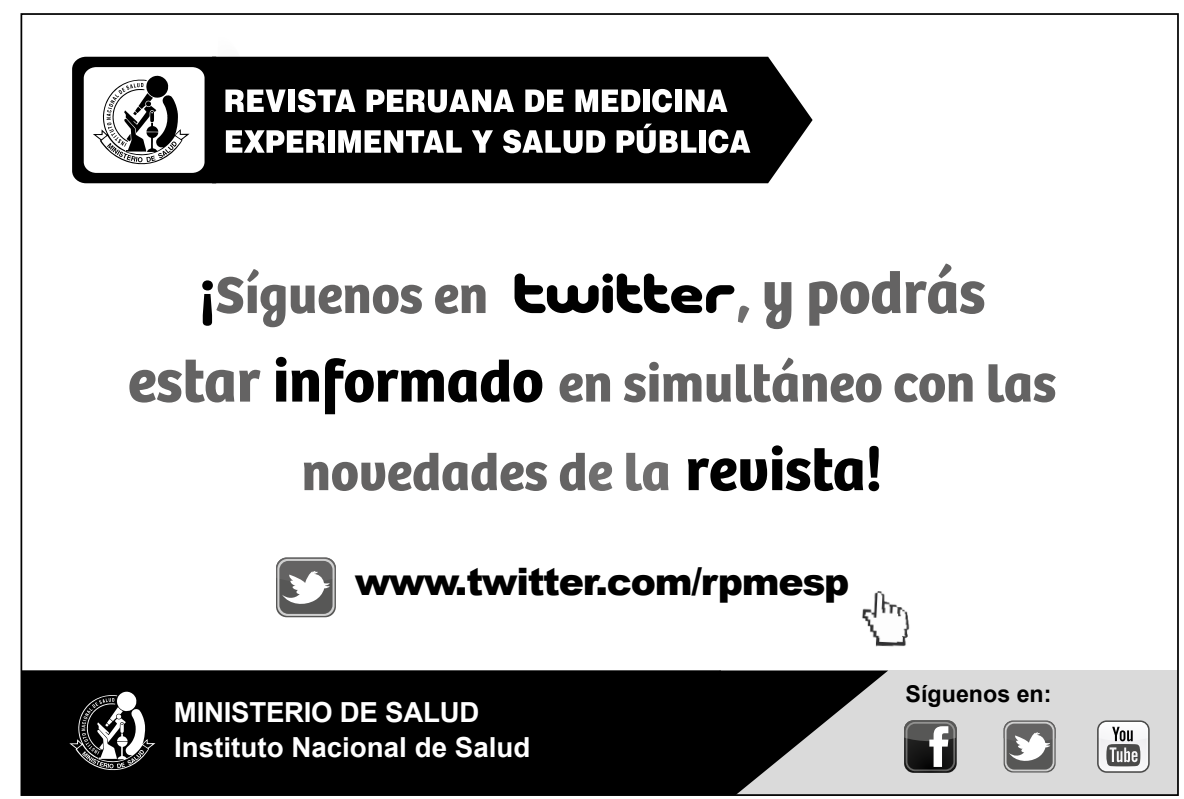

